\title{
Repelentes de insetos: recomendações para uso em crianças
}

\author{
Insect repellents: recommendations for use in children
}

\section{Germana Pimentel Stefani ${ }^{1}$, Antonio Carlos Pastorino ${ }^{2}$, Ana Paula B. M. Castro ${ }^{3}$, Angela Bueno F. Fomin ${ }^{3}$, Cristina Miuki A. Jacob ${ }^{4}$}

\section{RESUMO}

Objetivo: Apresentar uma revisão bibliográfica sobre o uso de repelentes de insetos em crianças, com ênfase especial na proteção contra mosquitos.

Fontes de dados: Realizou-se uma pesquisa bibliográfica nas bases de dados Pubmed e Lilacs, cujos artigos incluíam produtos comercialmente disponíveis no Brasil. Foram selecionados artigos publicados nos últimos dez anos em língua inglesa ou portuguesa, referentes a crianças, obtidos com as seguintes palavras-chave: "insect repellents", "children”, "mosquitoes", "N,N-dietil-metatoluamida (DEET)".

Síntese dos dados: Entre os repelentes tópicos, DEET, icaridina e óleo natural de eucalipto-limão apresentam, em concentrações adequadas, perfil de segurança favorável e são eficazes na prevenção de picadas de mosquitos em crianças e adultos. Em geral, são indicados para crianças acima de dois anos de idade. Medidas físicas são fundamentais para proteger lactentes jovens, especialmente menores de seis meses, com destaque para o uso de telas com permetrina.

Conclusões: Os mosquitos são vetores de doenças infectoparasitárias que acometem, anualmente, milhões de pessoas no mundo e causam milhares de mortes. O combate aos mosquitos inclui medidas ambientais e de proteção individual. O uso de repelentes tópicos para proteção individual da criança exige cuidados específicos e conhecimento quanto ao produto ideal para cada idade, especialmente quando consideradas sua eficácia e segurança.

Palavras-chave: repelentes de insetos; mosquitos; DEET; criança.

Instituição: Unidade de Alergia e Imunologia do Instituto da Criança do Hospital das Clínicas da Faculdade de Medicina da Universidade de São Paulo (ICr-HCFMUSP), São Paulo, SP, Brasil

'Médica Pediatra em estágio de Complementação Especializada em Alergia e Imunologia Pediátrica do ICr-HCFMUSP, São Paulo, SP, Brasil

${ }^{2}$ Doutor em Ciências pela FMUSP; médico assistente da Unidade de Alergia e Imunologia Pediátrica do ICr-HCFMUSP, São Paulo, SP, Brasil

${ }^{3}$ Mestre em Ciências pela FMUSP; médica assistente da Unidade de Alergia e Imunologia Pediátrica do ICr-HCFMUSP, São Paulo, SP, Brasil

${ }^{4}$ Professora-associada do Departamento de Pediatria da FMUSP; chefe da Unidade de Alergia e Imunologia Pediátrica ICr-HCFMUSP, São Paulo, SP, Brasil

\section{ABSTRACT}

Objective: To present a literature review about the use of insect repellents on children, highlighting the protection against mosquitoes.

Data sources: Pubmed and Lilacs databases were searched for articles in English and Portuguese published in the last ten years with the following key-words: "insect repellents", "children", "mosquitoes", "N,N-dietil-metatoluamida (DEET)".

Data synthesis: Concerning topical repellents, DEET, icaridine and natural oil of lemon eucalyptus are effective to prevent mosquito bites in children and adults and have favorable safety profile when used in appropriate concentration. They are generally recommended for children older than two years-old. Physical barriers are essential for infant protection, especially if they are six months old or less, with special interest in the permethrin-treated nets.

Conclusions: Mosquitoes are vectors of a variety of infectious and parasitic diseases which annually affect millions of people and cause thousands of deaths worldwide. The combat to mosquitoes includes individual and environmental measures. Topic repellents for children's individual protection demand specific handling attention and knowledge about the ideal product for each age, especially regarding their efficacy and safety.

Key-words: insect repellents; culicidae; DEET; children.

Endereço para correspondência:

Germana Pimentel Stefani

Unidade de Alergia e Imunologia do ICr

Avenida Doutor Enéas de Carvalho Aguiar, 647

CEP 05403-000 - São Paulo/SP

E-mail: gestefani@hotmail.com

Recebido em: 22/4/08

Aprovado em: 10/9/08 


\section{Introdução}

As doenças transmissíveis por insetos acometem milhões de pessoas em todo o mundo, causando milhares de mortes. Mosquitos são vetores de doenças para cerca de 700 milhões de habitantes ao ano, especialmente em países tropicais e subtropicais $^{(1-3)}$. Segundo a Organização Mundial de Saúde (OMS), somente a dengue acomete 50 milhões de pessoas no mundo anualmente, causando cerca de 24 mil mortes, e a malária, mais de 1 milhão de mortes por ano ${ }^{(4,5)}$. Estima-se que uma pessoa morra a cada 30 segundos por complicações relacionadas a picadas de insetos e que uma em cada 17 pessoas vivas morrerá de alguma dessas doenças ${ }^{(6,7)}$.

Epidemias de dengue vêm ocorrendo desde 1986 nos principais centros urbanos do Brasil, com cerca de 3 milhões de casos notificados. No período de 1990 a 2006, foram registrados 6.272 casos de Febre Hemorrágica da Dengue, com ocorrência de 386 óbitos. Em 2007, a incidência de dengue no país foi de 254,2 por 100 mil habitantes ${ }^{(5)}$. Dengue e febre amarela silvestre já acometeram mais de 50 mil pessoas somente no primeiro trimestre de 2008, com particular gravidade em crianças $^{(5)}$.

Somente as fêmeas dos mosquitos são hematófagas, ou seja, picam humanos para se alimentarem da albumina do sangue. Cada espécie tem seu habitat e ciclo próprio. Os artrópodes do gênero Aedes, por exemplo, são os vetores da dengue e febre amarela, têm maior atividade diurna e preferem ambientes abertos. Os do gênero Anopheles (transmissores de malária) preferem o amanhecer e o entardecer e frequentam ambientes tanto abertos quanto fechados ${ }^{(8)}$.

Além de serem vetores de doenças infecciosas, insetos podem ser causa de desconforto por suas picadas, especialmente em crianças com hipersensibilidade. Picadas de algumas espécies de mosquitos podem causar irritação local, prurido, pápulas, vesículas, estrófulo, infecção secundária local, celulite, dor, desconforto e distúrbios de sono, entre outros.

Há séculos, a humanidade busca maneiras de prevenir as picadas de insetos por meio de práticas naturais e/ou artificiais, na tentativa de evitar algumas doenças e também suas incômodas picadas ${ }^{(8,9)}$. Considerando que uma única picada de um inseto contaminado pode provocar alguma doença e que vacinas e quimioprofilaxia não estão ainda disponíveis para todos os casos, passa a ter importância o uso individual de repelentes ${ }^{(2)}$.

Este artigo tem como objetivo apresentar uma revisão bibliográfica sobre o uso de repelentes em crianças, com ênfase especial na proteção contra mosquitos, não sendo incluídos trabalhos sobre a proteção contra carrapatos, formigas, vespas, abelhas e outros artrópodes. A metodologia aplicada na presente revisão foi a pesquisa nas bases de dados Pubmed e Lilacs, considerando-se como critérios: artigos publicados nos últimos dez anos em língua inglesa ou portuguesa referentes a crianças e com as seguintes palavras-chave: "insect repellents", "children", "mosquitoes", "N,N-dietil-metatoluamida (DEET)". Consideraram-se relevantes 35 dos 164 artigos encontrados na Pubmed. A base de dados Lilacs disponibiliza 26 artigos sobre repelentes de insetos, dos quais somente cinco foram pertinentes à presente revisão. Incluiu-se, ainda, a recomendação da OMS quanto ao uso de repelentes.

Aqui serão referidos apenas os principais repelentes tópicos disponíveis comercialmente no Brasil. Informações técnicas acerca desses produtos foram obtidas em seus rótulos, no endereço eletrônico oficial do fabricante ou por contato telefônico com os serviços de atendimento ao consumidor. Serão discutidos os repelentes tópicos, físicos e ambientais, além dos aspectos de segurança e toxicidade e orientações de uso para a faixa etária pediátrica. Serão apresentados, também, os níveis de evidência já descritos sobre os repelentes e suas orientações pela entidade canadense Commitee to Advise on Tropical Medicine and Travel (CATMAT) ${ }^{(8)}$.

\section{Repelentes tópicos}

Repelentes tópicos podem ser sintéticos ou naturais. Eles atuam formando uma camada de vapor com odor repulsivo aos insetos sobre a pele ${ }^{(6,10)}$. As características ideais de um repelente seriam: repelir muitas espécies simultaneamente, ser eficaz por pelo menos oito horas, ser atóxico, ter pouco cheiro, ser resistente à abrasão e à água, cosmeticamente favorável e economicamente viável ${ }^{(6,7,10)}$.

Alguns fatores podem interferir na eficácia dos repelentes como a predisposição individual de acordo com substâncias exaladas pela pele (ácido lático, suor, $\mathrm{CO}_{2}$ ) e a existência de fatores de risco para picadas, entre os quais se destacam presença de eczema, sexo masculino, idade adulta, ingestão de álcool, vestimentas escuras, umidade, odor, clima quente e úmido, fragrâncias florais ${ }^{(2,10,11)}$. O sexo feminino, entretanto, é fator de risco para ineficácia do repelente, independentemente dos níveis de estradiol ${ }^{(12)}$, assim como a realização de atividades físicas moderadas ${ }^{(13)}$. Dessa forma, um repelente não protege igualmente todos os seus usuários ${ }^{(2,7)}$. Cada $10^{\circ} \mathrm{C}$ a mais na temperatura pode reduzir o tempo de proteção do repelente em até $50 \%{ }^{(3,7)}$. Um estudo de campo revelou que, em uma comunidade na qual os repelentes não são usados de 
forma universal, aqueles que não aplicam repelentes podem ter maior risco de picadas, já que passam a atrair os mosquitos repelidos por aqueles que usaram o produto ${ }^{(14)}$. Há várias formas de apresentação dos produtos: aerossol, gel, loção e spray. Na Tabela 1 estão expostos os principais produtos disponíveis no Brasil, com tempo de ação estimado.

- DEET (N,N-dietil-3-metilbenzamida ou N,N-dietil-mtoluamida) - grau de evidência $\mathrm{AI}^{(8)}$ : é o repelente mais eficaz atualmente disponível, sendo usado desde a década de 1950 em mais de 80 bilhões de aplicações ${ }^{(1,8,10)}$. Quanto maior a concentração da substância, mais longa é a duração da proteção (atingindo um platô em $35-50 \%)$, sem toxicidade relevante $\mathrm{e}^{(7,8,10,15)}$. Um estudo recente verificou que formulação com $4,75 \%$ de DEET confere proteção completa por 88 minutos; com 6,65\% de DEET a proteção dura 112 minutos; com 23,8\% de DEET a proteção é de 301 minutos em média, superior à proteção fornecida por óleo de soja e citronela ${ }^{(2)}$. Para uso habitual, altas concentrações não são necessárias. Deve-se, entretanto, considerar situações de altas temperaturas, umidade, chuva, área com grande chance de transmissão de doença e dificuldade de reaplicação $\operatorname{seriada}^{(7,8,10,15,16)}$. Formulações de liberação prolongada permitem boa proteção com menor quantidade de repelente. Para exposições demoradas, recomenda-se o uso de produtos com maior concentração e não a reaplicação seriada de produtos com menor quantidade de princípio ativo ${ }^{(7)}$.

A concentração máxima para uso em crianças é controversa: a Academia Americana de Pediatria (AAP) permite o uso de até $30 \%$ em maiores de dois anos ${ }^{(17)}$; a Sociedade Canadense de Pediatria (SCP) preconiza produtos com até $10 \%$ de DEET para crianças de seis meses a 12 anos $^{(8)}$ e autores franceses também sugerem concentrações de até $30 \%$ para crianças entre 30 meses e $12 \operatorname{anos}^{(9)}$. Na literatura, há um consenso de que se deve optar pela menor concentração efetiva e não são indicados para crianças com idade menor de dois a seis meses. No Brasil, a maioria dos produtos destinados a crianças e adultos contém DEET $<10 \%$ (Tabela 1).

- Icaridina ou KBR 3023 (1-piperidinecarboxylic acid, 2-(2-hydroxyethyl)-1-methylpropylester) - grau de evidência $\mathrm{AII}^{(8)}$ :é um novo e promissor repelente derivado da pimenta, indicado pela OMS para viajantes, juntamente com $\operatorname{DEET}^{(1,8,9)}$. Em concentração de $10 \%$ confere proteção por um período de três a cinco horas e, a $20 \%$, de oito a dez horas ${ }^{(6)}$. Sua ação é comparável a concentrações de $15-50 \%$ de DEET, mas permite reaplicações em intervalos maiores de tempo ${ }^{(6,8,18-20)}$. Estudo africano verificou que a potência do KBR 3023 contra o Anopheles gambiae

Tabela 1 - Repelentes disponíveis comercialmente no Brasil, concentrações e tempo de ação estimado

\begin{tabular}{|c|c|c|c|c|}
\hline Princípio ativo & $\begin{array}{l}\text { Produto (fabricante) e formas de } \\
\text { apresentação }\end{array}$ & Concentração $(\%)^{*}$ & $\begin{array}{c}\text { Idade } \\
\text { permitida* }\end{array}$ & $\begin{array}{c}\text { Tempo de ação } \\
\text { estimado* }\end{array}$ \\
\hline \multirow[t]{8}{*}{ DEET } & $\begin{array}{l}\text { Autan (Johnson Ceras) aerossol, } \\
\text { loção, spray }\end{array}$ & $6-9$ & $>2$ anos & Até 2 horas \\
\hline & OFF (Johnson Ceras) loção, spray & $6-9$ & $>2$ anos & Até 2 horas \\
\hline & OFF kids (Johnson Ceras) loção & $6-9$ & $>2$ anos & Até 2 horas \\
\hline & OFF (Johnson Ceras) aerossol & 14 & $>12$ anos & Até 6 horas \\
\hline & Super Repelex (Reckitt Benckis) & & $>12$ anos & Até 6 horas \\
\hline & spray, loção & 14,5 & & \\
\hline & aerossol & 11,05 & & \\
\hline & $\begin{array}{l}\text { Super Repelex kids gel (Reckitt } \\
\text { Benckis) }\end{array}$ & 7,34 & $>2$ anos & Até 4 horas \\
\hline \multirow[t]{3}{*}{ Icaridina } & Exposis adulto (Osler) gel, spray & 50 & $>12$ anos & Até 5 horas \\
\hline & Exposis Extreme (Osler) spray & 25 & $>10$ anos & Até 10 horas \\
\hline & Exposis infantil (Osler) spray & 25 & $>2$ anos & Até 10 horas \\
\hline IR3535 & $\begin{array}{l}\text { Loção antimosquito (Johnson \& } \\
\text { Johnson) }\end{array}$ & $* *$ & $>6$ meses & Até 4 horas \\
\hline Óleo de citronela & Citromim spray (Weleda) & 1,2 & $>2$ anos & Até 2 horas \\
\hline
\end{tabular}

*informações fornecidas pelo fabricante; **informação não fornecida pela empresa fabricante. 
não difere do DEET, mas contra o Aedes aegypti é de 1,1 a 2,0 vezes mais potente ${ }^{(21)}$. Após dez horas de exposição, é mais eficaz que o DEET e o IR $3535^{(22)}$. Há uma única marca disponível no Brasil e seu uso é recomendado para crianças acima de dois anos.

- IR 3535 (3-[N-acetyl-N-butyl]-aminopropionic acid ethyl ester): é um biopesticida sintético com estrutura química semelhante ao aminoácido alanina, disponível na Europa há mais de 20 anos. Em concentração de 20\%, é eficaz contra Anopheles e Aedes por um período de quatro a seis horas. Pode ser usado por gestantes, pois possui bom perfil de segurança. Na França, é recomendado apenas para crianças acima de 30 meses $^{(9)}$.

Estudo desenvolvido no Brasil comparou o IR3535 e o KBR 3023 a 10 e a 20\%, verificando-se uma média de proteção até a primeira picada de Aedes aegypti de seis horas (mínimo de cinco horas e 20 minutos), sem diferença significante entre os produtos ${ }^{(23)}$. Comparado ao DEET, o IR3535 foi igual ou superior na proteção contra duas espécies de flebótomos ${ }^{(24)}$. O tempo de proteção para Aedes aegypti foi similar ao DEET - cerca de três horas - com fórmulas a 10 e $20 \%$ de ambos os repelentes ${ }^{(25)}$. Há estudos, entretanto, que evidenciaram proteção média tão curta quanto seis a 23 minutos $^{(3)}$.

- Óleos naturais: os óleos são os mais antigos repelentes conhecidos e diversas plantas já foram utilizadas com tal propósito. Baseiam-se em essências de ervas, frutas cítricas, citronela, coco, soja, eucalipto, cedro, gerânio, hortelã e melissa, entre outros, e têm sido usados por séculos, com eficácia razoável ${ }^{(2)}$. Em geral, são altamente voláteis e, portanto, com efeito de curta duração ${ }^{(7)}$.

Em concentração de $2 \%$, o óleo de soja (Glycine max) conseguiu inibir picadas de Aedes por 94,6 minutos, sendo superior apenas ao DEET em concentração de 4,75\% $\%^{(2,26)}$. Acredita-se que esse óleo possua um efeito mecânico adicional de repelência. O óleo de citronela (Cymbopogon nardus), por ser extremamente volátil, confere proteção curta e variável de menos de 20 minutos a até duas horas, em concentrações de 5 a $100 \%{ }^{(8,27)}$. Recomenda-se a sua reaplicação a cada hora de exposição. O óleo de andiroba (Carapa guianensis) puro (100\%) mostrou, em um estudo brasileiro, efeito repelente discreto e muito inferior ao DEET 50\%, com tempo de proteção para a primeira picada de Aedes de 56 contra 3600 segundos respectivamente ${ }^{(11)}$.

O óleo de eucalipto-limão (Eucalyptus citriodora - Grau de evidência $\mathrm{AII}^{(8)}$ ) teve seu princípio ativo isolado re- centemente (p-menthane-3,8-diol: PMD). Em concentração de 30\%, é comparável ao DEET 20\% e confere proteção de até cinco horas, sendo o mais recomendado dos óleos naturais ${ }^{(2,8,29-31)}$. Todavia, há recomendação de reaplicações mais frequentes do que no caso do repelente sintético. A Sociedade Canadense de Pediatria coloca o óleo de eucalipto como segunda opção de repelente (a ser usado quando DEET é contraindicado e a icaridina não é disponível comercialmente no país $)^{(8)}$. A mistura de PMD e óleo de capim-limão (Cymbopogon citratus) foi superior ao DEET 15\% e conferiu proteção por mais de seis horas contra o Anopheles ${ }^{(32)}$.

\section{Repelentes físicos e ambientais}

São utilizados com o objetivo de evitar o contato com insetos e são sempre indicados como adjuvantes aos repelentes no cuidado das crianças. Lactentes jovens, especialmente menores de seis meses, são particularmente beneficiados por esse tipo de proteção, pois têm pouca mobilidade e ficam em locais restritos e de fácil proteção (berços, cercados, carrinhos de bebêe) ${ }^{(8,33)}$. Os repelentes tópicos, até o momento, não são indicados para uso em bebês menores de seis meses e devem ser aplicados com restrição entre seis meses e dois anos de idade ${ }^{(8)}$. Em áreas de alta densidade demográfica de mosquitos, deve-se garantir que as portas estejam bem vedadas, as janelas, fechadas e com telas ${ }^{(34)}$. Manter ambientes refrigerados com ar condicionado é uma forma altamente eficaz de manter mosquitos afastados do recinto, de tal forma que a OMS advoga que, em hotéis refrigerados, são desnecessárias outras medidas de proteção interna ${ }^{(1,34,35)}$. Mosquiteiros simples ou com aplicação de inseticidas (permetrina) são indicados na proteção noturna de adultos e crianças e na proteção diurna de lactentes jovens ${ }^{(1)}$. Tendas tratadas com inseticidas são altamente eficazes, seguras, duradouras e de acesso relativamente fácil ${ }^{(8,34,36,37)}$. Os poros das telas de mosquiteiros/tendas não devem ser maiores que $1,5 \mathrm{~mm}^{(1)}$. O uso combinado de repelente tópico e tela tratada com inseticida parece ter o melhor custo-benefício na prevenção de picadas ${ }^{(37,38)}$. O uso de vestimenta adequada (meias, blusas de mangas compridas e calças) é desejável, porém de baixa praticidade em um país de clima quente como o Brasil. Dá-se preferência a tecidos claros, devendo-se evitar cores muito chamativas ${ }^{(8)}$. Muitos repelentes podem ser aplicados sobre roupas (DEET, icaridina), conferindo proteção prolongada e diminuindo o uso tópico das 
substâncias. Há, inclusive, tecidos especiais que já vêm embebidos com repelentes, sendo mais indicados para a prática de esportes radicais em matas ${ }^{(1,8)}$.

O uso de repelentes ambientais deve seguir as orientações preconizadas para obtenção da ação esperada. Incensos e velas naturais só têm ação quando aplicados por horas contínuas e iniciados bem antes da exposição da pessoa ao ambiente. Velas e incensos de citronela não têm efeito repelente suficiente para que haja recomendação de seu uso isolado ${ }^{(7,34)}$. O uso da vela de andiroba por tempo prolongado ( 48 horas contínuas) em área com cerca de $27 \mathrm{~m}^{2}$ previne até $100 \%$ das picadas de Aedes aegypti ${ }^{(39)}$.

Inseticidas são substâncias que, além de repelir, matam os insetos. Há diversos derivados piretroides (permetrina e deltametrina os mais comuns) em várias formas de apresentação como spray, serpentinas, impregnados em roupas, mosquiteiros e telas, entre outros. O uso de mosquiteiros e telas com permetrina é altamente recomendado para crianças, sendo seguro também para gestantes ${ }^{(7)}$. A absorção percutânea é mínima ${ }^{(9,10)}$. As telas com permetrina podem ser usadas em ambientes externos para proteção de carrinhos de bebê, berços, redes, bebê-conforto, com produtos comerciais já desenvolvidos e adequados em tamanho para cada um desses objetos.

Aerossóis de inseticida têm o objetivo de matar os insetos presentes no local e prevenir a invasão domiciliar. Possuem efeito curto e devem ser aplicados em recintos fechados (de 10 a $20 \mathrm{~m}^{2}$ ) pelo menos duas horas antes de dormir. Recomenda-se o uso de aerossóis juntamente com serpentinas ou telas ${ }^{(1)}$. As serpentinas (elétricas ou não) são os inseticidas vaporizados mais conhecidos e, usualmente, contêm um piretroide como princípio ativo. Um aparelho é suficiente para proteção durante a noite em um quarto de tamanho habitual se o ambiente não for muito ventilado(1).

\section{Repelentes ineficazes}

Diversos dispositivos habitualmente utilizados são considerados ineficazes para proteção contra picadas de insetos. Os repelentes ultrassônicos não se mostraram eficazes em diversos estudos, assim como dispositivos elétricos luminosos com luz azul ${ }^{(7)}$. A luz atrai qualquer inseto, mas não previne definitivamente as picadas, visto que substâncias produzidas pelos indivíduos podem ser mais atraentes aos flebótomos do que a luz ${ }^{(7,39)}$. Bandagens embebidas em repelentes (pulseiras com DEET, por exemplo) também não são indicadas, uma vez que a repelência se dá por evaporação do princípio ativo sobre a pele e, comprovadamente, só protege até $4 \mathrm{~cm}$ da área aplicada ${ }^{(10)}$. Raquetes e outros instrumentos eletrocutores também não têm eficácia comprovada ${ }^{(8)}$.

Vários estudos, até o momento, não demonstraram eficácia suficiente para justificar o uso sistêmico de substâncias na tentativa de repelência pelo odor exalado no suor. Nesse item, incluem-se extratos de alho e vitamina B1, amplamente usada na prática clínica ${ }^{(2,3,8,28,39,40)}$.

\section{Segurança e toxicidade}

Há uma permanente preocupação da população quanto ao uso de repelentes em crianças. O DEET já é usado há mais de meio século por milhões de pessoas ${ }^{(2,26)}$. Estima-se que de 23 a 29\% das crianças americanas sejam expostas ao DEET ${ }^{(26,41)}$. Essa substância tem meia vida de 2,5 horas e a sua eliminação é predominantemente hepática, metabolizada pelo citocromo p450, podendo causar danos a materiais plásticos e a alguns tecidos ${ }^{(2,7,10,26)}$. Menos de 50 casos de efeitos tóxicos graves foram documentados na literatura desde 1960 e $75 \%$ desses resolveram-se sem sequelas ${ }^{(2)}$. As reações adversas são geralmente leves e incluem basicamente quadros dermatológicos como dermatite de contato, prurido, urticária, angioedema, erupção bolhosa e irritação conjuntival ${ }^{(10,41)}$. Há relatos de complicações neurológicas, com irritabilidade, fraqueza, tremores, confusão mental, agitação, atetose, convulsões, ataxia, encefalopatia, coma e três mortes por encefalite ${ }^{(10,26,42)}$. Desde 1992, não há novos relatos de efeitos graves em crianças, que parecem inclusive ter menor propensão a reações moderadas/ graves do que os adultos ${ }^{(26)}$. Vários consensos definem como seguro o uso de DEET em crianças, em concentrações de até $30 \%$, inclusive a OMS e a $\operatorname{AAP}^{(2,8,16,26,41,43)}$.

Para a maioria dos novos repelentes (icaridina e óleo de eucalipto, por exemplo), a recomendação de não aplicá-los em menores de dois anos não se dá por toxicidade, mas por falta de estudos nessa população ${ }^{(26,42)}$. Telas e mosquiteiros impregnados de permetrina têm boa segurança para crianças, inclusive bebês, não havendo contraindicação estabelecida $^{(8)}$.

O desenvolvimento de resistência a inseticidas, como aos piretroides, por algumas espécies de mosquitos tem sido motivo de preocupação recente. Em países africanos, essa resistência já começa a ter impacto sobre o sucesso dos programas de controle da malária baseados no uso de telas com inseticidas ou sprays domiciliares de piretroides e DDT ${ }^{(44,45)}$. Entretanto, o uso de telas, especialmente as 
tratadas com inseticidas, continuam sendo recomendadas, pois, comprovadamente, diminuem incidência e a gravidade da malária em crianças ${ }^{(46)}$. Ainda são desconhecidos os exatos efeitos sobre a saúde humana do aumento no número de mosquitos resistentes a inseticidas, mas há evidências de que o uso isolado de repelentes como DEET ou o uso combinado com inseticidas não-piretroides seja uma alternativa segura e eficaz ${ }^{(47,48)}$.

\section{Orientações de uso}

Há várias considerações a serem feitas sobre o uso seguro e correto dos repelentes em crianças. Em qualquer situação, é fundamental a leitura dos rótulos dos produtos. Deve-se, também, guardar a embalagem para acesso fácil no caso de possíveis intoxicações. É importante verificar a concentração do princípio ativo para se certificar de que é adequada para a idade da criança ${ }^{(9,10,41)}$.

De modo geral, os principais cuidados para uso de repelentes tópicos são:

a) Generosidade: a tendência natural é passar menos que o necessário.

b) Homogeneidade: a ação de um repelente limita-se a $4 \mathrm{~cm}$; a simples aplicação na bochecha não protege áreas adjacentes como o nariz ou o queixo.

c) Seletividade: todos os repelentes irritam as mucosas e, portanto, deve-se evitar passá-los nos olhos, boca e narinas e em áreas não expostas.

d) Repetição: deve-se aplicar o repelente conforme o tempo de exposição aos insetos e as orientações do fabricante ${ }^{(3)}$.

Repelentes devem ser aplicados apenas em áreas expostas, nunca sob roupas, e a pele em que foi aplicado o produto deve ser lavada com água e sabão quando findo o tempo de exposição. Evitar o uso em ambientes fechados (preferir outras medidas já mencionadas). Crianças não devem dormir com repelentes (use roupas compridas e mosquiteiros). A aplicação no rosto deve ser evitada e, se realizada, nunca aplicar o produto diretamente na face (aerossóis). O produto deve ser aplicado nas mãos do adulto e, então, no rosto da criança, evitando-se o contato com mucosas. Sempre lavar as mãos após as aplicações. Nunca permitir que crianças apliquem o repelente sozinhas ${ }^{(3,9,41)}$.

Reaplicações são necessárias conforme o tempo, o risco de exposição e a concentração do produto utilizado. Em locais de alta temperatura e umidade, a duração do efeito do repelente é menor e, portanto, reaplicações mais frequentes são necessárias. Se ocorrerem picadas antes do término do tempo de ação previsto pelo fabricante, o produto deve ser reaplicado. A SCP, entretanto, determina limites de reaplicações de DEET, conforme a idade da criança. Para crianças entre seis meses e dois anos, orienta-se uma aplicação diária; entre dois a 12 anos, até três aplicações diárias. Em caso de alto risco de transmissão de doenças e exposição prolongada, mais aplicações são permitidas, considerando-se o perfil de segurança do $\operatorname{DEET}^{(8,26)}$. Em caso de atividades na água, supõe-se que o produto será removido da pele e uma nova aplicação deverá ser realizada ${ }^{(16)}$.

Bloqueadores solares que contenham repelentes são desaconselhados, pois perdem efeito (a associação com DEET reduz eficácia em 34\% $)^{(3,8,35,42)}$. Além disso, filtros solares geralmente precisam ser reaplicados em intervalos mais curtos do que repelentes e o uso concomitante desses produtos pode aumentar a absorção sistêmica e a toxicidade deste último. Se necessário, aplicar primeiramente o protetor solar, aguardar a absorção completa do produto pela pele (cerca de 20 minutos) e, então, aplicar o repelente. Em caso de reaplicação, lavar o corpo com água e sabão e repetir o processo ${ }^{(8)}$. Evitar o uso de perfume e lavandas, pois são atrativos aos insetos ${ }^{(10)}$.

Estudo de campo conduzido com 301 responsáveis por crianças americanas revelou que pelo menos $1 / 3$ não lê os rótulos ou não segue as instruções presentes nos mesmos; há desatenção em relação à concentração dos produtos, havendo uma tendência ao uso de um único produto para toda a família, independentemente da idade, além da não-remoção do produto ao dormir em mais da metade das crianças, aumentando a chance de reações tóxicas ${ }^{(41)}$.

O Quadro 1 contém orientações pertinentes ao uso correto de repelentes e, na Tabela 2, estão disponíveis os níveis de evidências para recomendações de repelentes.

\section{Conclusões}

Crianças brasileiras, por viverem em um país tropical, têm alto risco de exposição a mosquitos, vetores ou não de doenças. É importante que o pediatra conheça adequadamente as medidas de prevenção às picadas de insetos e possa, assim, orientar corretamente os pais e responsáveis de crianças, especialmente que residam em ou viajem para áreas 
Quadro 1 - Resumo das recomendações gerais para o uso de repelentes em crianças

1. Não use repelentes tópicos em menores de dois meses de idade.

2. Leia todo o rótulo antes de aplicar o produto e conserve-o para consulta.

3. Aplique o produto conforme instruções do fabricante, somente nas partes do corpo permitidas. Não aplique próximo a produtos alimentícios.

4. Mantenha os repelentes fora do alcance de crianças e não permita sua auto-aplicação.

5. Evite o uso próximo a mucosas (boca, nariz, olhos, genitais) ou em pele irritada ou ferida. Para uso na face, primeiro aplique o produto nas mãos e então espalhe no rosto com cuidado.

6. Evite aplicação nas mãos das crianças. Sempre lave as mãos após aplicar o produto.

7. Use quantidade suficiente pra recobrir a pele exposta e evite reaplicações freqüentes.

8. Não use sob vestimentas.

9. Evite usar em conjunto com protetores solares.

10. Ao retornar a ambientes fechados, lave as áreas expostas ao produto com água e sabão.

11. Não durma com repelente no corpo, lave-se antes.

12. Se suspeitar de qualquer reação adversa ou intoxicação, lave a área exposta e entre em contato com serviço de intoxicação. Se necessário, procure serviço médico e leve consigo a embalagem do repelente.

Fonte: referências 8-10,15,41.

Tabela 2 - Nível de evidência para recomendação de métodos repelentes

\begin{tabular}{lc}
\hline Método repelente & Nível de evidência \\
\hline DEET $10-30 \%$ & A I \\
Icaridina $10-20 \%$ & A II \\
Óleo eucalipto-limão $10-30 \%$ & A II \\
Óleo de soja $2 \%$ & A II \\
Óleo de citronela $5-15 \%$ & E II \\
Tela tratada com piretroide & A I \\
Roupa tratada com permetrina & A II \\
\hline
\end{tabular}

Adaptado de CATMAT ${ }^{(8)}$.

de risco. Apesar da relevância e aplicabilidade do tema, há uma escassa produção científica, inclusive pelos países latinoamericanos, notadamente acometidos por doenças como malária e dengue.

DEET, icaridina e óleo de eucalipto-limão são eficazes na prevenção de picadas de mosquitos em crianças e adultos e apresentam perfil de segurança favorável. Medidas físicas são fundamentais para proteger lactentes jovens, especialmente menores de seis meses, destacando-se as telas com permetrina. Vários pontos devem ser observados pelos cuidadores para o uso correto de repelentes, propiciando-se, assim, proteção suficiente contra doenças transmitidas por mosquitos vetores. Em épocas de epidemias ou surtos de doenças transmitidas por vetores, as medidas de proteção individual expostas nesta revisão não devem ser tomadas como eficazes isoladamente. Medidas socioambientais coletivas devem ser sempre intensificadas e complementadas por estratégias que visem à não-proliferação e à erradicação dos mosquitos. 


\section{Referências bibliográficas}

1. World Health Organization [homepage on the internet]. International travel and health 2008 edition. [cited $2008 \mathrm{Apr}$ 10]. Available from: http://www. who.int/ith.

2. Fradin MS, Day JF. Comparative efficacy of insect repellents against mosquito bites. N Engl J Med 2002;347:13-8.

3. E-Medicine [homepage on the internet]. Fradin MS: Insect repellents [cited 2008 Apr 10]. Available from: http://www.emedicine.com/derm/topic540.htm.

4. World Health Organization [homepage on the Internet]. Vector-borne disease. [cited $2008 \mathrm{Apr} 10$ ]. Available from: http://www.who.int/heli/risks/ vectors/vector/en/

5. Centro de vigilância epidemiológica "Prof Alexandre Vranjac" - Secretaria de Estado da Saúde de São Paulo [homepage on the Internet]. Dengue em números [cited $2008 \mathrm{Apr}$ 10] Available from http://www.cve.saude.sp.gov.br/ htm/zoo/deng07_n2012.htm.

6. Bayrepel - the new active ingredient in AUTAN ${ }^{\circledR}$ [homepage on the internet]. [cited 2008 April 10] Available from http://www.autan.com/nqcontent. cfm?a_name=Info (see brochure)

7. Fradin MS. Mosquitoes and mosquito repellents: a clinician`s guide. Ann Intern Med 1998;128:931-40.

8. Commitee to Advise on Tropical Medicine and Travel (CATMAT). Statement on personal protective measures to prevent arthropod bites. Can Commun Dis Rep 2005;31:1-18.

9. Sorge F, Imbert P, Laurent C, Minodier P, Banerjee A, Khelfaoui F et al. Children arthropod bites protective measures: insecticides and repellents. Arch Pediatr 2007; 14:1442-50.

10. Brown M, Hebert A. Insect repellents: an overview. J Am Acad Dermatol 1997;36:243-9.

11. Miot HM, Batistella RF, Batista KA, Volpato DEC, Augusto LST, Madeira NG et al. Comparative study of the topical effectiveness of the andiroba oil (Carapa guianensis) and DEET 50\% as repellent for Aedes sp. Rev Inst Med Trop São Paulo 2004;46:253-6.

12. Golenda CF, Solberg VB, Burge R, Gambel JM, Wirtz RA. Gender-related efficacy difference to an extended duration formulation of topical $\mathrm{N}, \mathrm{N}$-diethylm-toluamide (DEET). Am J Trop Med Hyg 1999;60:654-7.

13. Schofield S, Tepper M, Gadawski R. Laboratory and field evaluation of the impact of exercise on the performance of regular and polymer-based DEET repellents. J Med Entomol 2007;44:1026-31.

14. Moore SJ, Davies CR, Hill N, Cameron MM. Are mosquitoes diverted from repellent-using individuals to non-users? Results of a field study in Bolivia. Trop Med Int Health 2007;12:532-9.

15. Sudakin DL, Trevathan WR. DEET: a review and update of safety and risk in the general population. J Toxicol Clin Toxicol 2003;41:831-9.

16. Pollack RJ. Repelling mosquitoes. N Engl J Med 2002;347:2-3.

17. American Academy of Pediatrics. Committee on Environmental Health. Follow safety precautions when using DEET on children. AAP News [serial on the internet]. 2003;22: 200399 [cited 2008 Apr 10]. Available from: http://aapnews. aappublications.org/cgi/content/full/e200399v1

18. Yap HH, Jahangir $\mathrm{K}$, Zairi J. Field efficacy of four insect repellent products against vector mosquitoes in a tropical environment. J Am Mosq Control Assoc 2000;16:241-4.

19. Debboun M, Strickman D, Solberg VB, Wilkerson RC, McPherson KR, Golenda $\mathrm{C}$ et al. Field evaluation of deet and a piperidine repellent against Aedes communis (Diptera: Culicidae) and Simulium venustum (Diptera: Simuliidae) in the Adirondack Mountains of New York. J Med Entomol 2000;37:919-23.

20. Yap HH, Jahangir K, Chong AS, Adanan CR, Chong NL, Malik YA et al. Field efficacy of a new repellent, KBR 3023, against Aedes albopictus (SKUSE) and Culex quinquefasciatus (SAY) in a tropical environment. J Vector Ecol 1998;23:62-8.

21. Badolo A, Ilboudo-Sanogo E, Ouédraogo AP, Constantini C. Evaluation of the sensitivity of Aedes aegypti and Anopheles gambiae complex mosquitoes to two insect repellents: DEET and KBR 3023. Trop Med Int Health 2004;9:330-4.
22. Costantini C, Badolo A, Ilboudo-Sanogo E. Field evaluation of the efficacy and persistence of insect repellents DEET, IR3535, and KBR 3023 against Anopheles gambiae complex and other Afrotropical vector mosquitoes. Trans R Soc Trop Med Hyg 2004;98:644-52.

23. Naucke TJ, Kropke R, Benner G, Schulz J, Wittern KP, Rose A et al. Field evaluation of the efficacy of proprietary repellent formulations with IR3535 and picaridin against Aedes aegypti. Parasitol Res 2007;101:169-77.

24. Naucke TJ, Lorentz S, Grunewald HW. Laboratory testing of the insect repellents IR3535 and DEET against Phlebotomus mascittii and $\mathrm{P}$ duboscqi (Diptera: Psychodidae). Int J Med Microbiol 2006;296(Suppl 40):230-2.

25. Cilek JE, Petersen JL, Hallmon CE. Comparative efficacy of IR3535 and deet as repellents against Aedes aegypti and Culex quinquefasciatus. J Am Mosq Control Assoc 2004;20:299-304.

26. Koren G, Matsui D, Bailey B. DEET-based insect repellents: safety implications for children and pregnant and lactating women CMAJ 2003;169:209-12.

27. Trongtokit Y, Rongsriyam Y, Komalamisra N, Apiwathnasorn C. Comparative repellency of 38 essential oils against mosquito bites. Phytother Res 2005;19:303-9.

28. Ives AR, Paskewitz SM; Inter-L\&S 101; Biology Interest Groups; Entomology Class 201. Testing vitamin $B$ as a home remedy against mosquitoes. J Am Mosq Control Assoc 2005;21:213-7.

29. Carroll SP, Loye J. PMD, a registered botanical mosquito repellent with deetlike efficacy. J Am Mosq Control Assoc 2006;22:507-14.

30. Erler F, Ulug I, Yalcinkaya B. Repellent activity of five essential oils against Culex pipiens. Fitoterapia 2006;77:491-4

31. Yang $P, M a Y$. Repellent effect of plant essential oils against Aedes albopictus. J Vector Ecol 2005;30:231-4.

32. Moore SJ, Darling ST, Sihuincha M, Padilla N, Devine GJ. A low-cost repellent for malaria vectors in the Americas: results of two field trials in Guatemala and Peru. Malar J 2007;6:101.

33. Evans MF. Which mosquito repellent works best? Can Fam Physician 2003;49:983-4.

34. Elston DM. Prevention of arthropod-related disease. J Am Acad Dermato 2004;51:947-54

35. Reiter P, Lathrop S, Bunning M, Biggerstaff B, Singer D, Tiwari T et al. Texas lifestyle limits transmission of dengue virus. Emerg Infect Dis 2003;9:86-9.

36. Edson F, Kayombo EJ. Knowledge on malaria transmission and its prevention among schoolchildren in Kyela District, south-western Tanzania. Tanzan Health Res Bull 2007;9:207-10.

37. Boulware DR, Beisang AA. Passive prophylaxis with permethrin-treated tents reduces mosquito bites among North American summer campers. Wilderness Environ Med 2005;16:9-15.

38. Rowland M, Freeman T, Downey G, Hadi A, Saeed M. DEET mosquito repellent sold through social marketing provides personal protection against malaria in an area of all-night mosquito biting and partial coverage of insecticide-treated nets: a case-control study of effectiveness. Trop Med Int Health 2004;9:343-50.

39. Saúde Total [homepage on the internet]. Miot HM: Atualização em repelentes de insetos [cited $2008 \mathrm{Apr}$ 10]. Available from: http://www.saudetotal.com/ artigos/dermatologia/repelentes.asp.

40. Rajan TV, Hein M, Porte P, Wikel S. A double-blinded, placebo-controlled trial of garlic as a mosquito repellant: a preliminary study. Med Vet Entomol 2005;19:84-9.

41. Menon KS, Brown AE. Exposure of children to Deet and other topically applied insect repellents. Am J Ind Med 2005;45:91-7.

42. Kendrick DB. Mosquito repellents and superwarfarin rodenticides - are they really toxic in children? Curr Opin Pediatr 2006;18:180-3.

43. Bell JW, Veltri JC, Page BC. Human Exposures to N,N-diethyl-m-toluamide insect repellents reported to the American Association of Poison Control Centers 1993-1997. Int J Toxicol 2002;21:341-52.

44. Irish SR, N'guessan R, Boko PM, Metonnou C, Odjo A, Akogbeto M et al. Loss of protection with insecticide-treated nets against pyrethroid-resistant Culex 
quinquefasciatus mosquitoes once nets become holed: an experimental hut study. Parasit Vectors 2008;1:17.

45. Corbel V, N'guessan R, Brengues C, Chandre F, Djogbenou L, Martin T et al. Multiple insecticide resistance mechanisms in Anopheles gambiae and Culex quinquefasciatus from Benin, West Africa. Acta Trop 2007;101:207-16.

46. Amodu OK, Olumese PE, Gbadegesin RA, Ayoola OO, Adeyemo AA. The influence of individual preventive measures on the clinical severity of malaria among Nigerian children. Acta Trop 2006;97:370-2
47. Pennetier C, Corbel V, Boko P, Odjo A, N'Guessan R, Lapied B et al. Synergy between repellents and non-pyrethroid insecticides strongly extends the efficacy of treated nets against Anopheles gambiae. Malar J 2007; 6:38

48. N'Guessan R, Rowland M, Moumouni TL, Kesse NB, Carnevale P. Evaluation of synthetic repellents on mosquito nets in experimental huts against insecticideresistant Anopheles gambiae and Culex quinquefasciatus mosquitoes. Trans R Soc Trop Med Hyg 2006;100:1091-7. 